\title{
A rare case of recurrent ovarian cancer with TPM3-NTRK1 gene rearrangement: A case report
}

\author{
YUTA ENDO $^{1}$, TAKAFUMI WATANABE ${ }^{1}$, MOTONOBU SAITO $^{2}$, KATSUHARU SAITO $^{2}$, REI SUZUKI ${ }^{3}$, \\ HIDEKI SANO $^{4}$, YUTAKA NATORI ${ }^{5}$, EISAKU SASAKI ${ }^{5}$, MAKIKO UEDA $^{1}$, NORIHITO KAMO ${ }^{1}$, \\ SHIGENORI FURUKAWA ${ }^{1}$, SHU SOEDA ${ }^{1}$, KOJI KONO ${ }^{2}$, SHIGEHIRA SAJI ${ }^{5}$ and KEIYA FUJIMORI ${ }^{1}$
}

Departments of ${ }^{1}$ Obstetrics and Gynecology, ${ }^{2}$ Gastrointestinal Tract Surgery, ${ }^{3}$ Gastroenterology, ${ }^{4}$ Pediatric Oncology and

${ }^{5}$ Medical Oncology, Fukushima Medical University School of Medicine, Fukushima 960-1295, Japan

Received December 9, 2021; Accepted February 8, 2022

DOI: $10.3892 / \mathrm{mco} .2022 .2523$

\begin{abstract}
NTRK gene fusion is rare in gynecological cancer. Entrectinib is a novel targeted drug, which is a potent inhibitor of TRK A, B and C. The present case report described a case of recurrent ovarian cancer with TPM3-NTRK1 rearrangement, which was detected by next-generation sequencing (NGS) and treated with entrectinib. A 56-year-old woman was diagnosed as having stage IV ovarian cancer with positive pleural fluid cytology. Neoadjuvant chemotherapy and interval debulking surgery, followed by chemotherapy, were performed. A total of 10 months after completion of chemotherapy, the disease recurred and the patient was treated with multimodal therapy for recurrence. DNA-based NGS detected TPM3-NTRK1 rearrangement and entrectinib therapy was initiated; however, the disease progressed despite 6 weeks of entrectinib administration, and 1 month after discontinuation of entrectinib, the patient died. After their death, immunohistochemistry with a pan-Trk monoclonal antibody was performed to determine the expression levels of TRK; however, immunohistochemistry was negative for TRK. In conclusion, the present case report described a rare case of recurrent ovarian cancer with TPM3-NTRK1 gene fusion, in which entrectinib was not effective. While NTRK gene fusion was detected by DNA-based NGS, immunohistochemistry was negative for TRK. These findings indicated that immunohistochemistry may be
\end{abstract}

Correspondence to: Dr Yuta Endo, Department of Obstetrics and Gynecology, Fukushima Medical University School of Medicine, 1 Hikarigaoka, Fukushima 960-1295, Japan

E-mail: yenyen@fmu.ac.jp

Abbreviations: IHC, immunohistochemistry; NGS, next-generation sequencing; OC, ovarian cancer; CA-125, cancer antigen 125; FIGO, International Federation of Gynecology and Obstetrics; $\mathrm{CT}$, computed tomography; SD, stable disease; BV, bevacizumab; $\mathrm{PD}$, progressive disease; $\mathrm{mAB}$, monoclonal antibody

Key words: NTRK gene fusion, TPM3-NTRK1, entrectinib, NGS, IHC, OC required for confirmation of TRK protein expression prior to entrectinib administration.

\section{Introduction}

NTRK gene fusions are consistently detected in rare types of cancers (secretory breast carcinoma, mammary analogue secretary carcinoma, congenital infantile fibrosarcoma, and congenital mesoblastic nephroma) and they are novel therapeutic targets across multiple tumor types (1-3). On the other hand, these gene fusions are rare in common adult cancers $(1,2)$. In gynecologic oncology, NTRK gene fusion is also rare, although there are several reports of uterine sarcoma with this fusion gene (4-7). A previous cohort study showed that TPM3-NTRK1 is most frequent in NTRK1 fusions across multiple histologies (2). Immunohistochemistry (IHC) staining, fluorescence in situ hybridization, reverse transcriptase polymerase chain reaction, DNA-based next-generation sequencing (NGS) and RNA-based NGS are used to identify patients with NTRK gene fusion cancer. Each method to detect $N T R K$ gene fusion has its own characteristics (3).

NTRK1, NTRK2 and NTRK3 encode TRKA, TRKB, and TRKC, respectively (1). Entrectinib is a potent inhibitor of TRKA, TRKB, TRKC, ROS1, and ALK, and is specifically designed to have systemic activity. In gynecologic oncology, treatment using entrectinib is rare because of the low frequency of NTRK fusions (1). Here, we report a case of recurrent ovarian cancer (OC) with TPM3-NTRK1 gene fusion, which was treated with entrectinib.

\section{Case report}

In September 2013, a 56-year-old woman was referred to Fukushima Medical University Hospital (Fukushima, Japan) with bilateral ovarian tumors, multiple disseminations in the peritoneum, bilateral pleural effusion, and multiple swellings of the pelvic and paraaortic lymph nodes. Her serum level of cancer antigen 125 (CA125) was elevated to 1,740 U/ml. She was diagnosed as having stage IV OC according to the International Federation of Gynecology and Obstetrics (FIGO) 1988 because pleural effusion cytology was positive. Paclitaxel $\left(175 \mathrm{mg} / \mathrm{m}^{2}\right)$ and carboplatin (area under the curve 6), TC 
therapy, were started as neoadjuvant chemotherapy. After four courses of chemotherapy, computed tomography (CT) revealed a reduction in tumor size. Interval debulking surgery including abdominal hysterectomy, bilateral salpingo-oophorectomy, omentectomy, and pelvic and paraaortic lymphadenectomy, was performed. Histopathological diagnosis was high-grade serous carcinoma. Following this surgery, another three courses of the same regimen were administered, and the patient achieved clinical complete response.

A total of 10 months after the last therapy, CT showed multiple disseminations around the liver. TC therapy was administered again. At the time of the third course, carboplatin-related hypersensitivity reaction occurred. After the third course, stable disease (SD) was shown. Thus, TC therapy was converted to TP therapy $\left(135 \mathrm{mg} / \mathrm{m}^{2}\right.$ paclitaxel and $75 \mathrm{mg} / \mathrm{m}^{2}$ cisplatin). After three courses of TP therapy, SD was maintained. As the disseminations were located only around the liver, partial hepatectomy was performed. At that time, postoperative chemotherapy was not administered as there was no detectable disease in the abdominal cavity.

A total of 5 months after the surgery, CT showed multiple lesions in the peritoneum. Therefore, TP therapy with $15 \mathrm{mg} / \mathrm{m}^{2}$ bevacizumab (BV) was started. At the ninth course of this chemotherapy, cisplatin-related hypersensitivity reaction occurred. After the ninth course, CT showed progressive disease (PD). Subsequently, chemotherapy with pegylated liposomal doxorubicin with $\mathrm{BV}$, gemcitabine with $\mathrm{BV}$, and nogitecan with BV was administered. However, the tumors remained; mesentery dissemination resection was performed.

A total of 8 months after mesentery dissemination resection, CT showed multiple peritoneal lesions. Subsequently, weekly paclitaxel, oral etoposide, weekly nedaplatin and gemcitabine were administered in this order, however, none of the regimens were effective. Microsatellite stability was detected in specimens from the mesentery dissemination resection.

In August 2019, because there was no more standard therapy, FoundationOne ${ }^{\circledR}$ CDx (Foundation Medicine, Cambridge, MA), which is DNA based NGS and covers 324 genes, was performed based on the patient's archival tumor tissue from the mesentery dissemination resection. This revealed a missense variant of TP53 (c.731G $>$ A) and TPM3-NTRK1 rearrangement between somewhere around exon 2-3 of TPM3 (pos1='chr1:156844554-156844771', pos2='chr1:154155588-154155822') and exon 11 of NTRK1 (NM_002529). Oral entrectinib (600 mg/day) was started after discussing with experts. A total of 6 weeks after initiation of entrectinib, the patient's serum CA125 level elevated to $4,360 \mathrm{U} / \mathrm{ml}$, which was $1,712 \mathrm{U} / \mathrm{ml}$ before initiation of entrectinib, and CT revealed progression of liver metastasis (Fig. 1). Adverse events during entrectinib administration comprised grade 2 dysgeusia. A total of 1 month after discontinuation of entrectinib, the patient died from disease progression (Fig. 2).

After the patient's death, IHC staining with a pan-Trk monoclonal antibody (mAB) clone EPR17341 (Abcam, Cambridge, MA) was performed to assess TRKA, TRKB, and TRKC expression as previously described (8). This $\mathrm{mAB}$ clone is most commonly used and has been investigated thoroughly. In addition, this $\mathrm{mAB}$ clone reacts with
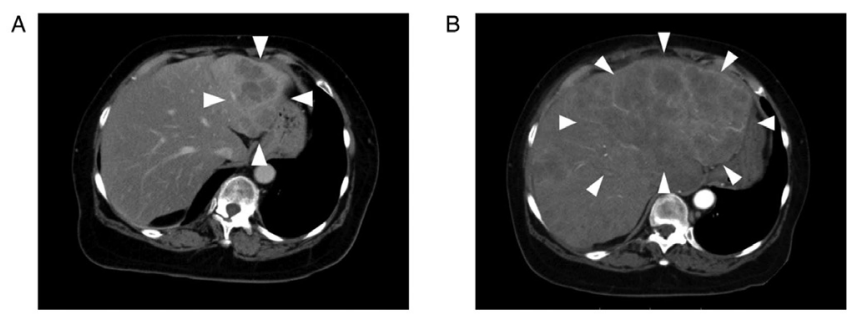

Figure 1. Computed tomography of liver metastasis; arrow heads show the liver metastasis lesion. (A) Before administration of entrectinib. (B) A total of 6 weeks after beginning administration of entrectinib.

a conserved proprietary peptide from the C-terminus of TRKA, TRKB and TRKC, and is therefore reactive to any oncogenic NTRK fusion (3). IHC was negative for all specimens from the primary site, as well as the first and second recurrent sites (Fig. 3).

\section{Discussion}

Here we presented a case of recurrent OC with TPM3-NTRK1 rearrangement. Additionally, the present case demonstrates the discrepancy between gene rearrangement detected by NGS and protein expression. This discrepancy may be an indicator for predicting the ineffectiveness of entrectinib for cancers with NTRK rearrangement detected by NGS.

In the current case, NGS revealed TPM3-NTRK1 rearrangement and a missense variant of TP53. There are few approved therapies for TP53 variants, although almost all cases of ovarian high-grade serous carcinoma $(95 \%)$ have somatic TP53 variants (9). On the other hand, NTRK fusions are oncogenic drivers and novel targets. Doebele et al (1) reported the safety and activity of entrectinib in adult patients with advanced or metastatic NTRK fusion-positive cancers across three clinical trials (ALKA-372-001, STARTRK-1 and STARTRK-2). In these trials, only one ovarian cancer patient was included. They showed that the objective response rate, which included complete response and partial response, was $57 \%$ (95\% CI 43.2-70.8). The median duration of response was 10 months (95\% CI 7.1 to not estimable) and the percentage of PD was only $7 \%$. However, the characteristics of cases with PD remained unclear in their report (1).

In the present case, entrectinib was administered because NGS revealed TPM3-NTRK1 rearrangement and entrectinib was recommended after a discussion among experts. However, this novel target drug was ineffective. TRK protein was not expressed as a result of IHC testing with a pan-Trk mAB clone (EPR17341). A previous study reported that gene fusions involving $N T R K 1,2$, and 3 and their partner genes result in a constitutive activation or overexpression of TRK receptors, potentially leading to oncogenesis (10). Additionally, other reports have shown that pan-Trk IHC yielded a sensitivity of 75-95.2\%, and a specificity of 92-100\% and that the sensitivity of pan-Trk IHC for TRKA was $96.2 \%(3,8,11,12)$. Pan-Trk IHC is a reliable screening method for the detection of NTRK gene fusions based on these data. Moreover, pan-Trk IHC is used to assess rapidly assess malignancies which may harbor possible NTRK gene fusions in order to determine eligibility of patients for 


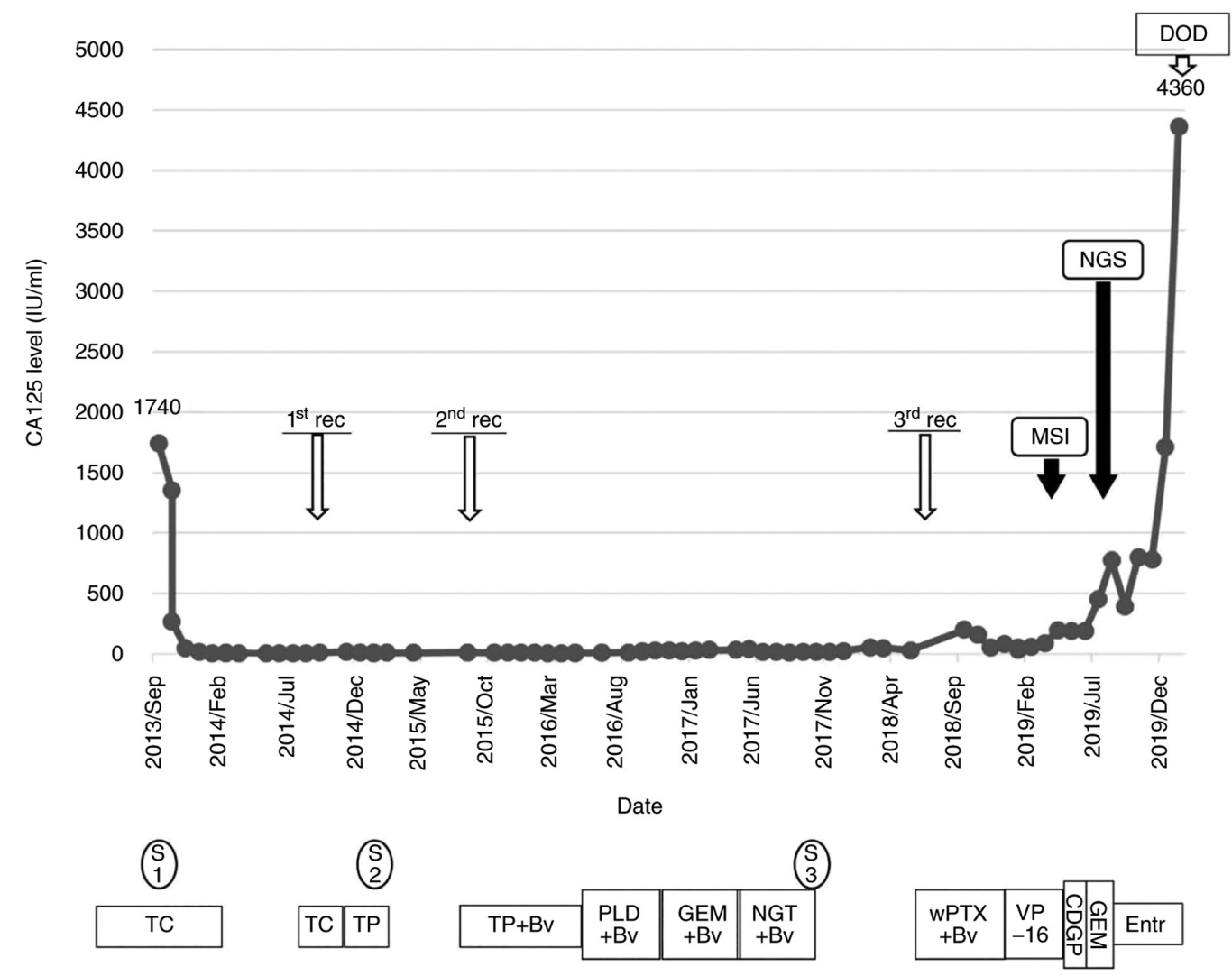

Figure 2. Clinical flowchart of the patient. CA125, cancer antigen-125; MSI, microsatellite instability test; NGS, next-generation sequencing; DOD, dead of disease; rec, recurrence; S, surgery; S1, abdominal hysterectomy, bilateral salpingo-oophorectomy, omentectomy, and pelvic and paraaortic lymphadenectomy; S2, partial hepatectomy; S3, mesentery dissemination resection; TC, paclitaxel and carboplatin; TP, paclitaxel and cisplatin; BV, bevacizumab; PLD, pegylated liposomal doxorubicin; GEM, gemcitabine; NGT, nogitecan; wPTX, weekly paclitaxel; VP-16, etopocide; CDGP, nedaplatin; Entr, entrectinib.
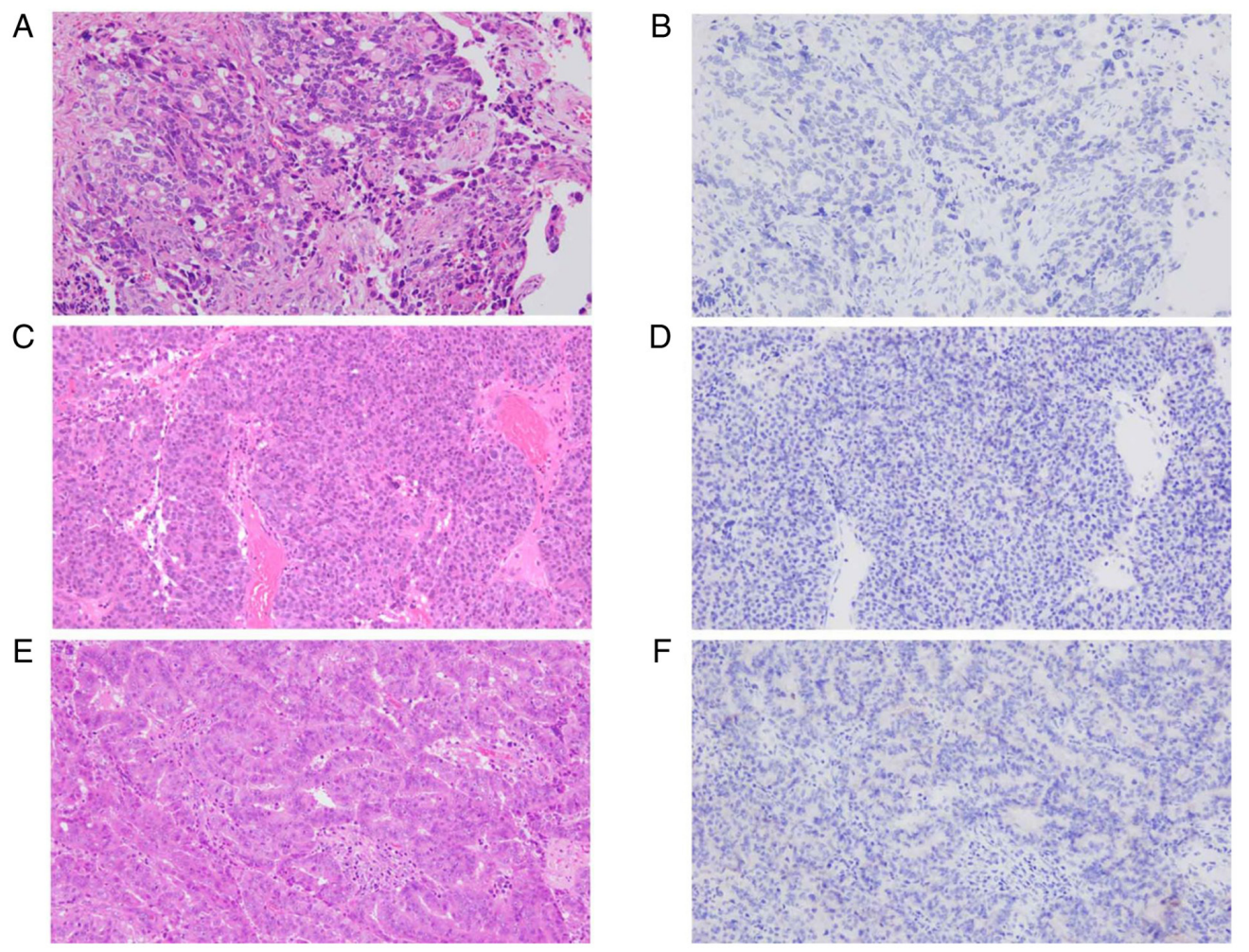

Figure 3. Histopathological and immunohistochemical features (magnification, x100). Immunohistochemistry was negative for pan-TRK in all specimens. (A and B) Interval debulking surgery specimen (tumor resection following neoadjuvant chemotherapy). (C and D) Liver dissemination specimen (resection for the first recurrence). (E and F) Mesentery dissemination specimen (recurrence for the second recurrence). (A, C and E) Hematoxilin and eosin staining. (B, D and F) Immunohistochemical stain for pan-Trk. 
targeted therapy with TRK inhibitors (8). However, it should be considered that there are $N T R K$ rearrangements which are found to be negative by IHC, and can only be detected by NGS, such as in the present case.

Drilon et al (13) reported the efficacy of larotrectinib, which is a selective inhibitor of TRKA, TRKB and TRKC. In their study, six of an initial 55 patients showed primary resistance to larotrectinib. Three of the six patients had tumor material available for pan-Trk IHC, by which TRK protein expression was not detected in all three. This indicated that the rearrangements detected by NGS were false positives or that the identified fusion genes were not expressed at the protein level (13). It is considered that entrectinib has the same characteristics as larotrectinib with regard to discrepancy between gene fusion and protein expression, as observed in the current case, and that this finding may be a key to predict the ineffectiveness of entrectinib for cancers with $N T R K$ rearrangement detected by NGS.

To the best of our knowledge, this is the first case report of OC with $N T R K$ rearrangement. It is known that a small percentage of common adult cancers carry fusions of NTRK genes (2). Large cohort studies revealed that the frequency of NTRK gene fusions was $0.25 \%$ of general cancers $(2,12)$. Therefore, physicians have few chances to experience this molecular characteristic; however, they should be aware of the pitfall that TRK protein may not express even if NGS shows NTRK rearrangement.

In conclusion, we here presented a rare case of recurrent OC with TPM3-NTRK1 fusion. Physicians may need to be aware of the discrepancy of DNA rearrangement and protein expression, and IHC may be required for confirmation of TRK protein expression before entrectinib administration.

\section{Acknowledgements}

Not applicable.

\section{Funding}

No funding was received.

\section{Availability of data and materials}

The datasets used and/or analyzed during the current study are available from the corresponding author on reasonable request.

\section{Authors' contributions}

YE, TW and MS drafted the manuscript. YE, TW, RS, HS, YN, ES, MU, NK, SF, SSo, SSa and KF contributed to the patient management and manuscript editing. MS, KS and KK performed immunohistochemical staining. YE and TW confirm the authenticity of all the raw data. All authors have read and approved the final manuscript.

\section{Ethics approval and consent to participate}

\section{Patient consent for publication}

Written informed consent was obtained from the patient for publication of this case report and the accompanying images.

\section{Competing interests}

The authors declare that they have no competing interests.

\section{References}

1. Doebele RC, Drilon A, Paz-Ares L, Siena S, Shaw AT, Farago AF, Blakely CM, Seto T, Chow BC, Tosi D, et al: Entrectinib in patients with advanced or metastatic NTRK fusion-positive solid tumours: Integrated analysis of three phase 1-2 trials. Lancet Oncol 21: 271-282, 2020.

2. Getalica Z, Xiu J, Swensen J and Vranic S: Molecular characterization of cancers with NTRK gene fusions. Mod Pathol 32: 147-153, 2019.

3. Solomon JP, Benayed R, Hechtman JF and Ladanyi M: Identifying patients with NTRK fusion cancer. Ann Oncol 30 (Suppl 8): viii16-viii22, 2019.

4. Chiang S, Cotzia P, Hyman DM, Drilon A, Tap WD, Zhang L, Hechtman JF, Frosina D, Jungbluth AA, Murali R, et al: NTRK fusions define a novel uterine sarcoma subtype with features of fibrosarcoma. Am J Surg Pathol 42: 791-798, 2018.

5. Rabban JT, Devine WP, Sangoi AR, Poder L, Alvarez E, Davis J, Rudzinski E, Garg K and Bean GR: NTRK fusion cervical sarcoma: A report of three cases, emphasising morphological and immunohistochemical distinction from other uterine sarcomas, including adenosarcoma. Histopathology 77: 100-111, 2020.

6. Hodgson A, Pun C, Djordjevic B and Turashvili G: NTRKrearranged cervical sarcoma: Expanding the clinicopathologic spectrum. Int J Gynecol Pathol 40: 73-77, 2021.

7. Croce S, Hostein I and McCluggage WG: NTRK and other recently described kinase fusion positive uterine sarcomas: A review of a group of rare neoplasms. Genes Chromosomes Cancer 60: 147-159, 2021

8. Hechtman JF, Benayed R, Hyman DM, Drilon A, Zehir A, Frosina D, Arcila ME, Dogan S, Klimstra DS, Ladanyi M and Jungbluth AA: Pan-Trk immunohistochemistry is an efficient and reliable screen for the detection of NTRK fusions. Am J Surg Pathol 41: 1547-1551, 2017.

9. Boyarskikh UA, Gulyaeva LF, Avdalyan AM, Kechin AA, Khrapov EA, Lazareva DG, Kushlinskii NE, Melkonyan A, Arakelyan A and Filipenko ML: Spectrum of TP53 mutations in BRCA1/2 associated high-grade serous ovarian cancer. Front Oncol 10: 1103, 2020.

10. Khotskaya YB, Holla VR, Farago AF, Mills Shaw KR, Meric-Bernstam $\mathrm{F}$ and Hong DS: Targeting TRK family proteins in cancer. Pharmacol Ther 173: 58-66, 2017.

11. Rudzinski ER, Lockwood CM, Stohr BA, Vargas SO, Sheridan R, Black JO, Rajaram V, Laetsch TW and Davis JL: Pan-Trk immunohistochemistry identifies NTRK rearrangements in pediatric mesenchymal tumors. Am J Surg Pathol 42: 927-935, 2018.

12. Solomon JP, Linkov I, Rosado A, Mullaney K, Rosen EY, Frosina D, Jungbluth AA, Zehir A, Benayed R, Drilon A, et al: NTRK fusion detection across multiple assays and 33,997 cases: Diagnostic implications and pitfalls. Mod Pathol 33: 38-46, 2020.

13. Drilon A, Leatsch TW, Kummar S, DuBois SG, Lassen UN, Demetri GD, Nathenson M, Doebele R, Farago AF, Pappo AS, et al: Efficacy of larotrectinib in TRK fusion-positive cancers in adults and children. N Engl J Med 378: 731-739, 2018.

Not applicable. 